\title{
Selective confinement of macroscopic long-lifetime exciton and trion populations
}

\author{
M. D. Fraser, H. H. Tan, and C. Jagadish \\ Department of Electronic Materials Engineering, Research School of Physics and Engineering, The Australian National University, \\ Canberra, ACT 0200, Australia
}

(Received 14 May 2011; revised manuscript received 26 October 2011; published 27 December 2011)

\begin{abstract}
Confinement of cold macroscopic particle ensembles opens avenues to study many-body coherent states of matter in a controlled environment. We create pure macroscopic cold long-lifetime trion and exciton gases and arbitrary mixtures spatially confined with fabricated electrostatic potentials on GaAs/AlGaAs coupled quantum well structures. The population densities are readily controlled by electric field and laser power. In the exciton phase, we observe a strong contraction of the exciton mode area at high densities and small traps, indicating the onset of exciton quantum degeneracy, linking and establishing an experimental system in which to study potential macroscopic quantum phases of cold trion gases.
\end{abstract}

DOI: 10.1103/PhysRevB.84.245318

PACS number(s): 71.35.Pq, 71.35.Lk, 67.85.Pq

Excitons $^{1}$ are the most suitable bosonic quasiparticle for conducting atomic-like experiments in a solid-state environment, having properties analogous to a low-mass finitelifetime hydrogen atom. Charged excitons, known as trions, ${ }^{2}$ are also stable excited states of semiconductors amenable to manipulation. ${ }^{3}$ Macroscopic quantum states of bosonic atoms are created as Bose-Einstein condensates (BECs), ${ }^{4}$ while charged fermionic electron gases exhibit phases, such as a charged Fermi liquid, ${ }^{5}$ or a macroscopic coherence via Cooper pairing in the superconductivity phase. ${ }^{6}$ There exists a continuous transition between the $\mathrm{BEC}$ and the Cooper pair mediated Bardeen-Cooper-Schrieffer (BCS) phases, known as the BEC-BCS crossover. ${ }^{7,8}$ We present a novel system where interconversion between bosonic exciton and charged fermionic trion populations is readily achieved and controlled in an environment where an exciton BEC-like state is expected, ${ }^{4,9}$ opening the new possibility of the creation of macroscopic quantum states of trions.

We consider the confinement and manipulation of cold, long-lifetime populations of both exciton and trion gases in the same structure, where the relative population of each species can be controlled by an applied electrical field and optical excitation. The long-lifetime stable exciton gas is achieved with two-dimensional spatially indirect excitons $X_{I}$ in a coupled quantum well (CQW) structure, ${ }^{10}$ which has long been considered a strong candidate for a BEC-like state, ${ }^{11-13}$ though challenging notably due to disorder and low transition temperatures. ${ }^{14}$ The prospects for condensation are promising, given recent reports of exciton quantum degeneracy, ${ }^{15-17}$ an important precursor to observing exciton condensation and of long coherence lengths. ${ }^{18}$ Although our experiments are conducted in the steady state, the exciton lifetime is considerably greater than the trion formation time, offering the unique possibility to study dynamics of trapped mixtures of Bose and charged Fermi particles. We further establish the experimental possibility of quantum phases of a trapped trion gas by demonstrating signatures of quantum degeneracy in the exciton phase in such a trap.

We study two molecular-beam-epitaxy (MBE) grown GaAs/ $/ \mathrm{Al}_{0.3} \mathrm{Ga}_{0.7}$ As CQW samples $\left(S_{n}\right.$ and $\left.S_{p}\right)$ with well widths of $t_{w}=8 \mathrm{~nm}\left(S_{n}\right)$ and $t_{w}=12 \mathrm{~nm}\left(S_{p}\right)$ and tunnel barrier thickness $t_{b}=4 \mathrm{~nm}$. The CQW of $S_{n}$ is symmetrically embedded in a $n^{+}-i-n^{+}$structure $\left(n_{e} \sim 10^{18} \mathrm{~cm}^{-3}\right)$ with intrinsic thickness $t_{i}=320 \mathrm{~nm}$, while in $S_{p}$, the CQW is asymmetrically situated $50 \mathrm{~nm}$ from the substrate in a $p^{+}-i-n^{+}$structure $\left(n_{h} \sim 10^{19} \mathrm{~cm}^{-3}\right)$ with intrinsic thickness $t_{i}=328 \mathrm{~nm}$. The key difference is the use of $n^{+}-i-n^{+}$and $p^{+}-i-n^{+}$heterostructures which limit sample current and background carrier concentrations. As such, $S_{n}$ is used for exciton-trion mixture experiments and $S_{p}$ for studying dense exciton phases, although excitons and trions can be observed in both structures. Applying a perpendicular electric field $F_{\perp}$ to this sample breaks the $z$ symmetry of the wells, separating the electrons into one well, the holes into the other. ${ }^{10,14,19}$ The resulting neutral indirect excitons $X_{I}^{0}$ have an interwell recombination transition [Fig. 1(b)] in contrast to the intrawell transition of direct excitons $X_{D}^{0}$ [Fig. 1(a)]. Due to the effective band tilting of the quantum confined Stark shift, the $X_{I}^{0}$ luminescence is linearly red shifted in response to $F_{\perp}$, and enhanced significantly over that of a single $\mathrm{QW}$, putting $X_{I}^{0}$ in a high-field seeking state.

Spatial variations in this applied electric field $F_{\perp}$ are used to create potential minima and localize the excitons laterally. The $n^{+} / p^{+}$cap layer is patterned into a circular shape to spatially modify $F_{\perp}$ within the CQW layer. Electrostatic trapping of $X_{I}^{0}$ has previously been considered, ${ }^{20-23}$ however we additionally add a thick $(\sim 250 \mathrm{~nm}) \mathrm{SiO}_{2}$ layer to the surface of the regions outside the trapping area, which due to its low dielectric permittivity (in contrast with $\mathrm{Al}_{x} \mathrm{Ga}_{1-x} \mathrm{As}$ ), effectively screens the electric field from these regions, allowing electrical contacts to the trapping regions without the associated trap losses. Further, indium-tin-oxide (ITO) is used as a transparent surface electrical contact (with good Ohmic contacts to $n^{+} / p^{+}$-GaAs, and transparency $>95 \%$ ), deposited uniformly to the entire sample. The design advantages include low exciton losses from in-plane field ionization, ${ }^{24}$ and small electric fields relative to the ionization field are able to generate deep trapping potentials, with almost arbitrarily complex time and space dependent profiles. The structure transparency also allows for largely distortion-free imaging of the spatial exciton and trion luminescence profiles, an important requirement for studying spatial properties of the exciton and trion gases.

Trions are normally observed only in tightly confined quantum dots ${ }^{25}$ as microscopic populations, or as unconfined gases in modulation-doped quantum wells ${ }^{26}$ or resonant tunneling diodes. ${ }^{27}$ In this trap structure, we observe the efficient 

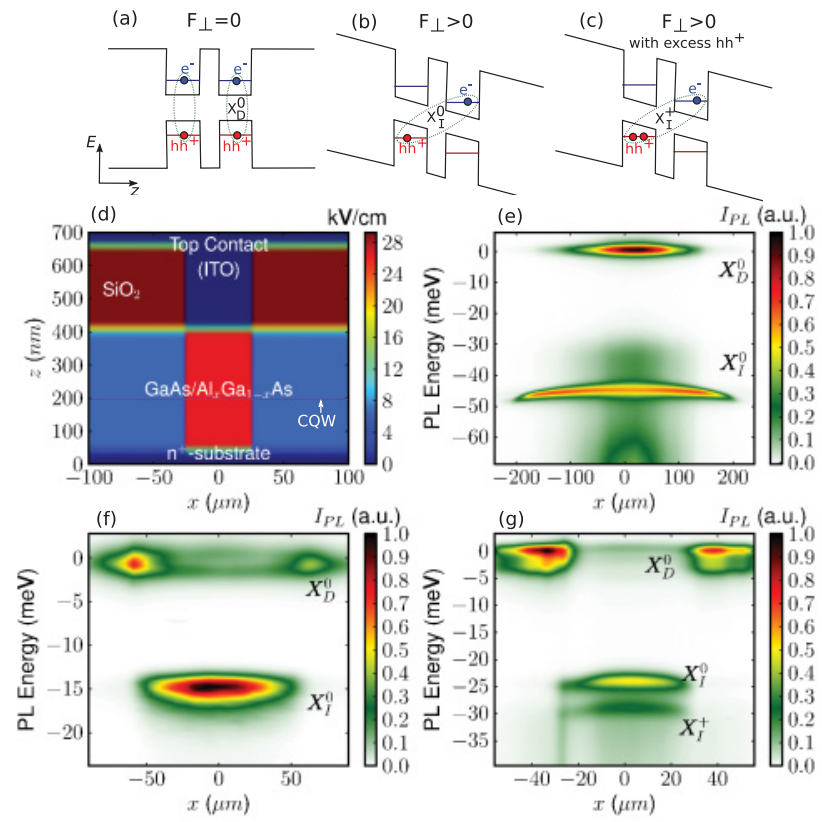

FIG. 1. (Color online) Schematic CQW band diagrams for the (a) $X_{D}^{0}$, (b) $X_{I}^{0}$, and (c) $X_{I}^{+}$under differing bias/excitation conditions. (d) Simulated electric field cross-sectional (through $y$ axis) profile shows $\mathbf{F}(x, z)$ is well confined to the trap region despite being uniformly applied to the sample surface. Experimental measurements of (e) untrapped excitons $\left(X_{D}^{0}\right.$ and $\left.X_{I}^{0}\right)$ showing long-range diffusion of $X_{I}^{0}$, (f) selective trapping of $X_{I}^{0}$ within the linear regime, and (g) simultaneous trapping of $X_{I}^{0}$ and $X_{I}^{+}$within the nonlinear regime.

formation of a controlled macroscopic trion population, expected to have long radiative lifetimes and be thermalized close to the lattice temperature. Charged species (trions and free carriers) also feel the spatially localized electric field $F(x, y)$ (within the CQW plane), which attracts one charge while repelling the other depending on the gate voltage $V_{G}$ polarity. The simultaneous trapping of excitons and free carriers (if injected in sufficient densities) enhances the formation rate and stability of exciton-trions. To create trions our structure relies on charged carrier injection by photoexcitation (above the $\mathrm{Al}_{0.3} \mathrm{Ga}_{0.7} \mathrm{As}$ barrier) ${ }^{28}$ and background free carriers/leakage current. The heavier mass of holes leads to more efficient capture than electrons resulting in a spatial region within the laser excitation profile containing a steady-state population of excitons and heavy holes. We observe a strong asymmetry in the prevalence of trion formation with $V_{G}$ polarity and thus assign the consistently easier direction to heavy-hole trions $X_{I}^{+}$[Fig. 1(c)].

The typical cross-sectional electric field amplitude profile $F(x, z)$ is shown in Fig. 1(d), with dielectric, semiconductor, and conducting layers indicated. The electric field is localized within the semiconductor region below the trapping contact, though the entire sample surface is at a fixed potential. Both $X_{I}^{0}$ and $X_{I}^{+}$are observed [Figs. 1(f) and 1(g)], with steady-state relative populations controlled through pumping power $P_{l}$, electric field $F_{\perp}$, or trap size. The $X_{I}^{0}$ and $X_{I}^{+}$populations are studied using an imaging microphotoluminescence ( $\mu \mathrm{PL})$ setup with samples immersed in a liquid He cryostat at $T=$ 1.4 K. Laser illumination $(\lambda=633 \mathrm{~nm} \mathrm{HeNe} C W)$ and PL collection are both via the same aspheric objective lens ( $f=$ $4 \mathrm{~mm}$, numerical aperture $=0.6$ ), also immersed in the liquid $\mathrm{He}$, where under superfluid He conditions clear images result. The PL image is projected onto the entrance slit of a $750 \mathrm{~mm}$ imaging spectrometer, measuring the spectral distribution of a one-dimensional (1D) spatial trap cross section yielding the effective trap depth and exciton distribution.

At a sample position without trapping potentials, we observe cold long-lifetime excitons with radially diffusive motion exhibiting $X_{I}^{0}$ diffusion lengths far exceeding the laser diameter [Fig. 1(e)]. At excitation powers below the Mott transition, the $X_{I}$ experience a mean-field energy blue shift with increasing density. ${ }^{29}$ The rapid out-diffusion of excitons creates a radially varying density profile, evidenced by a chevron-shaped $E-x$ profile, ${ }^{24}$ indicating a larger density and mean-field interaction energy at the laser excitation center. The mean-field blue shift of the overall cloud is small because of out-diffusion and lack of population buildup, though this changes dramatically in trapped measurements.

In patterned regions of the sample at $V_{G}>0$, the $X_{I}^{0}$ cloud is completely confined to the trap area [Fig. 1(f)], while excitation outside the trap results in only $X_{D}^{0}$ (the laser is larger than the trap in this measurement). Under experimental conditions of larger laser powers, smaller trap sizes, or larger voltages, a $X_{I}^{+}$population is observed (a $2-3 \mathrm{meV}$ lower energy peak to the $X_{I}^{0}$ population) within the trap [Fig. $\left.1(\mathrm{~g})\right]$. The $X_{I}^{0}$ trapped cloud has a higher density at the trap center, and the $X_{I}^{+}$population is usually slightly more centrally localized, given lower free carrier populations at the trap edges. No clear $X_{D}^{+}$populations (i.e., untrapped trions) are observed.

Like $X_{I}^{0}$ in untrapped samples, the trapped $X_{I}^{0}$ and $X_{I}^{+}$ clouds also Stark shifts to lower energy with increasing $F_{\perp}$. Figure 2(a) shows a sequence of measurements of a

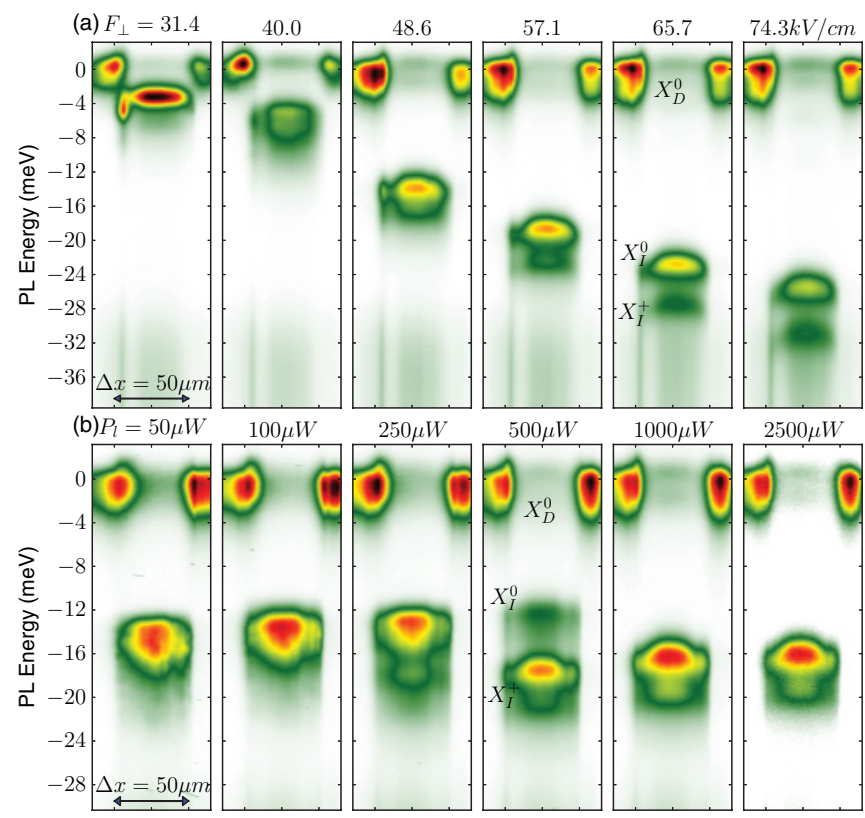

FIG. 2. (Color online) Experimental measurements of a spectrally resolved $50 \mu \mathrm{m}$-diameter trap cross section showing trapped $X_{I}^{0}$ and $X_{I}^{+}$formation as a function of (a) increasing applied electric field $F_{\perp}$ and (b) laser excitation power $P_{l}$. 
$50 \mu \mathrm{m}$-diameter trap as a function of $F_{\perp}$, where the transition from $X_{D}^{0}$ to $X_{I}^{0}\left(F_{X_{D}^{0} \rightarrow X_{I}^{0}} \approx 25 \mathrm{kV} / \mathrm{cm}\right)$ and the emergence of a $X_{I}^{+}\left(F_{X_{I}^{0} \rightarrow X_{I}^{+}} \gtrsim 50 \mathrm{kV} / \mathrm{cm}\right)$ peak are observed. Unlike in untrapped samples however, where the Stark shift is approximately linear, the trapped samples display a roughly piecewise nonlinear energy evolution. At fields just above the $X_{D}^{0} \rightarrow X_{I}^{0}$ transition, the $X_{I}^{0}$ Stark shift is roughly linear though shallower than in untrapped samples due to the confinementinduced density enhancement. However, the appearance of a $X_{I}^{+}$population corresponds to a simultaneous reduction in the Stark-shift slope. At large voltages, ionization of both $X_{I}^{0}$ and $X_{I}^{+}$occurs with rapid red shifts and population loss.

Figure 2(b) shows the evolution of the trapped population at a fixed voltage in a $50 \mu \mathrm{m}$ trap as $P_{l}$ is increased, exhibiting a rapid and complete macroscopic trion population appearance. At intermediate $P_{l}$ the $X_{I}^{0}$ peak completely transforms to a $X_{I}^{+}$peak, red shifted by $\Delta E \approx 2.5 \mathrm{meV}$. Whereas in the $F_{\perp}$-controlled transition, the trap depth slowly enhances the exciton and free-carrier population, the increase of $P_{l}$ induces a much more dramatic increase. In modulation-doped QW experiments, only a partial population transformation is usually observed; however, in these exciton-trion traps, we observe dynamical conversion of $100 \%$ of the $X_{I}^{0}$ population to $X_{I}^{+}$.

The energy dependence of the $X_{I}^{0}$ and $X_{I}^{+}$peaks as a function of $F_{\perp}$ and $P_{l}$ [Figs. 3(a) and 3(b)] can be used to ascertain the approximate densities of the various particles to model the mechanisms by which populations are controlled. Based on the observation of comparable luminescence intensities, particularly across the $X_{I}^{0}-X_{I}^{+}$transition [Fig. 2(b)], we assume the $X_{I}^{+}$and $X_{I}^{0}$ gases have similar radiative lifetimes and population densities (given the complete population transfer in some measurements). The system of trapped $X_{I}$ and a freecarrier mixture can then be understood by considering a simple
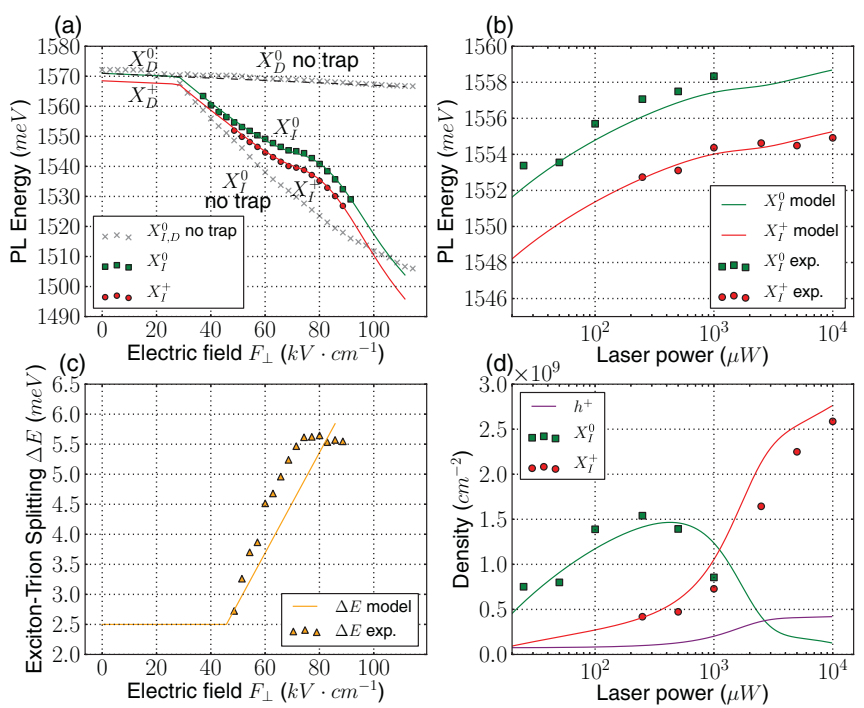

FIG. 3. (Color online) For a $50 \mu$ m-diameter trap, (a) and (b) show the energy evolution of the trapped $X_{I}^{0}$ and $X_{I}^{+}$populations (at the trap center) with $F_{\perp}$ and $P_{l}$ following from Figs. 2(a) and 2(b), respectively, and fitted with theoretical data from a chemical rate equation model, (c) shows $X_{I}^{0}-X_{I}^{+}$splitting and (d) shows the absolute $X_{I}^{0}$ and $X_{I}^{+}$populations estimated from experiments and fit with the numerical model. rate equation model ${ }^{30}$ where, under $\mathrm{CW}$ pumping and static bias, all populations are in a steady-state chemical equilibrium $X_{I}^{0}+h^{+} \rightleftharpoons X_{I}^{+}$. This model contains two variables for each state $i$, namely, the energy $E_{i}$ and density $n_{i}$, which are a function of pumping intensity $P_{l}$ and electric field $\mathbf{F}$. The direct exciton energy $E_{X_{D}^{0}}$ is taken from experiment. A density dependent blue shift is observed to control the $X_{I}^{0}$ energy of the form $\Delta E_{X}^{M F}\left(n_{X_{I}^{0}}\right)=4 \pi e^{2} d n_{X_{I}^{0}} / \epsilon$ derived from dipole-dipole mean-field interaction contributions, ${ }^{29}$ with dipole strength $d \approx 20 \mathrm{~nm}$. The density of trions at a particular $\mathbf{F}$ and $P_{l}$ resulting from a population of excitons $n_{X^{0}}$ and heavy holes $n_{h h}$ is then given by

$$
\begin{aligned}
n_{X^{+}}\left(\mathbf{F}, P_{l}\right)= & \frac{2 \pi \hbar^{2}}{k_{B} T} n_{h h}\left(\mathbf{F}, P_{l}\right) n_{X^{0}}\left(\mathbf{F}, P_{l}\right) \\
& \times \frac{m_{X^{0}}+m_{h h}}{m_{X^{0}} m_{h h}} e^{-\Delta E / k_{B} T},
\end{aligned}
$$

where $m_{i}$ and $n_{i}$ are the masses and densities, respectively, of species $i \in\left\{X^{0}, h h\right\} .{ }^{30}$ The $X_{I}^{+}$density thus depends notably on the injection of excitons and holes and on the $X_{I}^{0}$ and $X_{I}^{+}$energy splitting $\left(\Delta E=E_{X^{+}}-E_{X^{0}}\right) . n_{h}\left(\mathbf{F}, P_{l}\right)$ is difficult to estimate as it is sourced by contributions from background doping, optical injection, and lateral charge imbalance/electrical injection through $n^{+}-i-n^{+}$junction leakage. However, assuming that for a given $F$ and $P_{l}$, the total exciton population $\left(n_{X}=n_{X_{I}^{0}}+n_{X_{I}^{+}}\right.$) is an exponential function of $F$ above the $X_{D}^{0} \rightarrow X_{I}^{0}$ transition and logarithmic in $P_{l}$, specifically $n_{X}\left(F_{\perp}\right) \propto \exp \left(C_{2}\left(F_{\perp}-F_{X_{D}^{0} \rightarrow X_{I}^{0}}\right)\right)+\log _{10}\left(P_{l}\right)$ (where $C_{2}$ is a fitting parameter $), n_{X^{+}}\left(\mathbf{F}, P_{l}\right)$ and $E_{X^{+}}$are successfully predicted for the parameter space investigated experimentally. $\Delta E$ [Fig. 3(c)] is a complex combination of the trion binding energy $\left(E_{B}^{X_{I}^{+}} \approx-2.5 \mathrm{meV}\right),{ }^{31}$ exciton interactions, Stark shifts, and binding energy screening effects, estimated by

$$
\begin{aligned}
E_{X^{+}}= & E_{X_{D}^{0}}-C_{1 a}\left(F_{\perp}-F_{X_{D}^{0} \rightarrow X_{I}^{0}}\right)+E_{B}^{X_{I}^{+}} \\
& +E_{M F}\left(n_{X_{I}^{0}}, n_{X_{I}^{+}}\right)+C_{1 b}\left(F_{\perp}-F_{X_{I}^{0} \rightarrow X_{I}^{+}}\right) .
\end{aligned}
$$

The trion energy will shift with the electric field at a different rate to neutral excitons $\left(C_{1 a}\right)$ because of its charge (with rate $C_{1 b}$ ), which occurs after a turn-on field $F_{X_{I}^{0} \rightarrow X_{I}^{+}}$ corresponding to the point where the population of holes becomes large enough (due to either trapping or pumping buildup mechanisms) to facilitate a chemical equilibrium of trions. Approximate Coulomb interactions between trions and holes $E_{C}^{M F}\left(n_{X_{I}^{+}}\right)=\left(e^{2} / 4 \pi \epsilon\right) \sqrt{n_{X_{I}^{+}}}$are also included when the trion population becomes significant. The experimental data is closely fit with this theory, though the ionization region of $\Delta E$ is neglected.

This model accurately describes the energy evolution, trion splitting, and the $X_{I}^{0} \rightarrow X_{I}^{+}$transition where all data for a single trap size are fit with a single set of parameters. Exciton and trion densities [Fig. 3(d)] estimated from this model and from experimental mean-field shifts also agree, falling comfortably below the ionization density $n_{\text {ion }} \sim 10^{11} \mathrm{~cm}^{-2}$. We find, in general, that smaller trapping diameters enhance the observed effects, namely, faster trap-induced population buildup of both $X_{I}^{0}$ and $X_{I}^{+}$with $V_{G}$ and $P_{l}$. Finer control over 

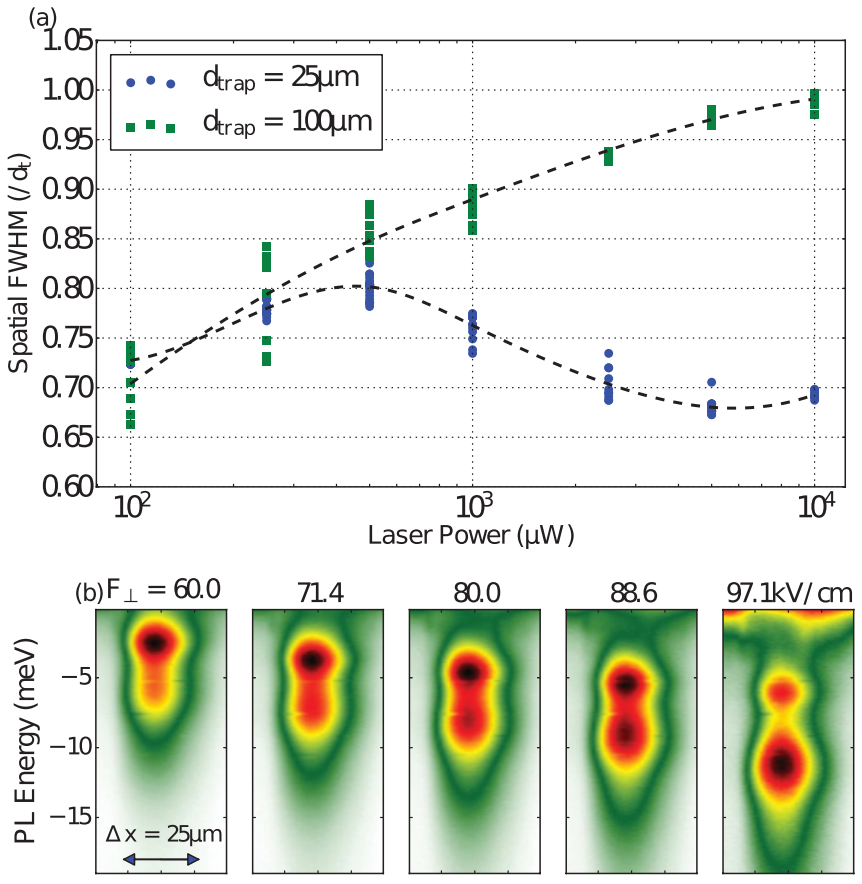

FIG. 4. (Color online) (a) Evolution of the width of a spatially trapped $X_{I}^{0}$ profile (with respect to trap diameter) for trap diameters of 100 and $25 \mu \mathrm{m}$, which show contrasting population buildup behavior. The dashed lines are fit to the weighted average. (b) Observation of $X_{I}^{+}$populations in a $25 \mu \mathrm{m}$ trap at elevated voltages and laser power $P_{l}=2.5 \mathrm{~mW}$ in sample $S_{p}$. The trapped $X_{I}^{0}$ linewidths are taken only in the exciton phase.

the relative populations could be achieved with a two-color excitation experiment, ${ }^{26}$ using both an above band laser (for $X_{I}^{0}$ and $X_{I}^{+}$) and a $X_{D}$-resonant laser (only $X_{I}^{0}$ ).

To establish an experimental link between the cold trapped trion gas and a potential macroscopic quantum phase of excitons, we present evidence for the onset of quantum degeneracy in the trapped exciton phase. In the $n^{+}-i-n^{+}$sample $S_{n}$, the trion phase is usually observed prior to sufficient exciton density accumulation. However, in the $p^{+}-i-n^{+}$sample $S_{p}$, with significantly lower free-carriers and current, the trap occupation is almost entirely $X_{I}^{0}$ at similar voltages, thus being more ideal for investigations of a cold pure exciton gas. An abruptly narrowing spatial distribution ${ }^{16}$ is considered partial evidence of quantum degeneracy onset, though not previously observed in a controllable trapped gas. For (lithographically defined) trapping diameters of 100 and $25 \mu \mathrm{m}$, the (spectrally integrated) spatial profile full width at half maximum (FWHM) evolution with pumping power is plotted in Fig. 4(a). The many points at each laser power represent different voltages, covering the effect of trap-depth induced population buildup.

With increased density, interaction energies broaden the spatial profile of a repulsively interacting trapped gas, and the corresponding monotonic increase in spatial width is observed in the $100 \mu \mathrm{m}$ trap. The $25 \mu \mathrm{m}$ trap is markedly different, showing first expansion, followed by a contraction and subsequent re-expansion of the exciton cloud diameter, the changes being of order $15 \%$, indicating that in small Stark traps at a temperature of $1.2 \mathrm{~K}$ and densities below the ionization limit, a quantum degenerate state of excitons is reached

The lower current and free carrier population in sample $S_{p}$ allowed for the study of gases largely consisting of $X_{I}$, and the measurements in Fig. 4(a) were collected in this phase. However, even in this sample, when both the laser power and the applied voltage are large enough to generate sufficient free carriers, conversion between $X_{I}^{0}$ and $X_{I}^{+}$can also be observed. Figure 4(b) shows the (voltage-induced) evolution of a $X_{I}^{+}$-peak from the $X_{I}^{0}$-peak when excited at $P_{l}=2.5 \mathrm{~mW}$ and at voltages elevated beyond that of the pure exciton gas. The voltage-induced conversion between populations is gradual, as in sample $S_{n}$; however to better study the nature of the transition between a degenerate $X_{I}^{0}$ gas and a $X_{I}^{+}$gas, a sample with simultaneously large achievable $X_{I}^{0}$ density and a large window of $X_{I}^{+}$occupation is advantageous. This might be more readily achieved using a fine-tuned combination of resonant and off-resonant excitations and/or light doping in a similar structure to sample $S_{p}$.

Macroscopic quantum phases of trions have not been readily discussed in the literature. A charged Fermi liquid might be expected (provided sufficient binding energy), though a mechanism (to parallel Cooper pairing) for driving coherence in trion gases is not yet established, possibly due to the lack of an experimental system. Here we have demonstrated the selective confinement of cold long-lifetime trions and excitons in arbitrary mixtures, wherein the exciton phase exhibits signatures of quantum degeneracy. Not only is a cold pure trion gas observed, but the long lifetime is expected to permit dynamic exciton-trion conversion, thus opening the new possibility to explore potential macroscopic quantum states in a trion gas, and novel bosonic-charged fermionic crossover physics in a controlled environment. ${ }^{32}$

The authors acknowledge the Australian Research Council for financial support.
${ }^{1}$ C. F. Klingshirn, Semiconductor Optics (Springer, Berlin, 2005).

${ }^{2}$ H. Buhmann, L. Mansouri, J. Wang, P. H. Beton, N. Mori, L. Eaves, M. Henini, and M. Potemski, Phys. Rev. B 51, 7969 (1995).

${ }^{3}$ D. Sanvitto, F. Pulizzi, A. J. Shields, P. Christianen, S. N. Holmes, M. Y. Simmons, D. A. Ritchie, J. C. Maan, and M. Pepper, Science 294, 837 (2001).
${ }^{4}$ A. Griffin, D. W. Snoke, and S. Stringari, Bose-Einstein Condensation (Cambridge University Press, Cambridge, 1995).

${ }^{5}$ D. Pines and P. Nozières, Theory of Quantum Liquids, Volume I: Normal Fermi Liquids (Addison-Wesley, Reading, MA, 1994).

${ }^{6}$ A. J. Leggett, Quantum Liquids: Bose Condensation and Cooper Pairing in Condensed-Matter Systems (Oxford University Press, Oxford, 2006). 
${ }^{7}$ M. Randeria, J. M. Duan, and L. Y. Shieh, Phys. Rev. Lett. 62, 981 (1989).

${ }^{8}$ M. Greiner, C. A. Regal, and D. S. Jin, Nature (London) 426, 537 (2003).

${ }^{9}$ S. A. Moskalenko and D. W. Snoke, Bose-Einstein Condensation of Excitons and Biexcitons: and Coherent Nonlinear Optics with Excitons (Oxford University Press, Oxford, 2000).

${ }^{10}$ Y. J. Chen, E. S. Koteles, B. S. Elman, and C. A. Armiento, Phys. Rev. B 36, 4562 (1987).

${ }^{11}$ L. V. Butov, A. Zrenner, G. Abstreiter, G. Böhm, and G. Weimann, Phys. Rev. Lett. 73, 304 (1994).

${ }^{12}$ V. Negoita, D. W. Snoke, and K. Eberl, Phys. Rev. B 60, 2661 (1999).

${ }^{13}$ L. V. Butov, C. W. Lai, A. L. Ivanov, A. C. Gossard, and D. S. Chemla, Nature (London) 92, 47 (2002).

${ }^{14}$ L. V. Butov and A. I. Filin, Phys. Rev. B 58, 1980 (1998).

${ }^{15}$ L. V. Butov, A. L. Ivanov, A. Imamoglu, P. B. Littlewood, A. A. Shashkin, V. T. Dolgopolov, K. L. Campman, and A. C. Gossard, Phys. Rev. Lett. 86, 5608 (2001).

${ }^{16}$ C. W. Lai, J. Zoch, A. C. Gossard, and D. S. Chemla, Science 303, 503 (2004).

${ }^{17}$ R. Rapaport, G. Chen, and S. Simon, Appl. Phys. Lett. 89, 152118 (2006).

${ }^{18}$ S. Yang, A. T. Hammack, M. M. Fogler, L. V. Butov, and A. C. Gossard, Phys. Rev. Lett. 97, 187402 (2006).

${ }^{19}$ J. E. Golub, K. Kash, J. P. Harbison, and L. T. Florez, Phys. Rev. B 41, 8564 (1990).

${ }^{20}$ R. Rapaport, G. Chen, S. Simon, O. Mitrofanov, L. Pfeiffer, and P. M. Platzman, Phys. Rev. B 72, 075428 (2005).
${ }^{21}$ A. T. Hammack, N. A. Gippius, S. Yang, G. O. Andreev, L. V. Butov, M. Hanson, and A. C. Gossard, J. Appl. Phys. 99, 066104 (2006).

${ }^{22}$ G. Chen, R. Rapaport, L. N. Pffeifer, K. West, P. M. Platzman, S. Simon, Z. Vörös, and D. Snoke, Phys. Rev. B 74, 045309 (2006).

${ }^{23}$ A. A. High, A. K. Thomas, G. Grosso, M. Remeika, A. T. Hammack, A. D. Meyertholen, M. M. Fogler, L. V. Butov, M. Hanson, and A. C. Gossard, Phys. Rev. Lett. 103, 087403 (2009).

${ }^{24}$ Z. Vörös, R. Balili, D. W. Snoke, L. Pfeiffer, and K. West, Phys. Rev. Lett. 94, 226401 (2005).

${ }^{25}$ D. V. Regelman, E. Dekel, D. Gershoni, E. Ehrenfreund, A. J. Williamson, J. Shumway, A. Zunger, W. V. Schoenfeld, and P. M. Petroff, Phys. Rev. B 64, 165301 (2001).

${ }^{26}$ N. N. Sibeldin, M. L. Skorikov, and V. A. Tsvetkov, Nanotechnology 12, 591 (2001).

${ }^{27}$ F. J. Teran, L. Eaves, L. Mansouri, H. Buhmann, D. K. Maude, M. Potemski, M. Henini, and G. Hill, Phys. Rev. B 71, 161309 (2005).

${ }^{28}$ V. B. Timofeev, A. V. Larionov, M. Grassi Alessi, M. Capizzi, A. Frova, and J. M. Hvam, Phys. Rev. B 60, 8897 (1999).

${ }^{29}$ M. Stern, V. Garmider, V. Umansky, and I. Bar-Joseph, Phys. Rev. Lett. 100, 256402 (2008).

${ }^{30}$ C. R. L. P. N. Jeukens, P. C. M. Christianen, J. C. Maan, D. R. Yakovlev, W. Ossau, V. P. Kochereshko, T. Wojtowicz, G. Karczewski, and J. Kossut, Phys. Rev. B 66, 235318 (2002).

${ }^{31}$ A. S. Bracker, E. A. Stinaff, D. Gammon, M. E. Ware, J. G. Tischler, D. Park, D. Gershoni, A. V. Filinov, M. Bonitz, F. M. Peeters, and C. Riva, Phys. Rev. B 72, 035332 (2005).

${ }^{32}$ H. Saarikoski, S. M. Reimann, A. Harju, and M. Manninen, Rev. Mod. Phys. 82, 2785 (2010). 